\title{
Fatigue in patients with Parkinson's disease
}

\author{
Kazuo Abe*, Mayako Takanashi and \\ Takehiko Yanagihara \\ Department of Neurology, Osaka University Graduate \\ School of Medicine, Japan
}

\begin{abstract}
Purpose: Fatigue is a complaint frequently encountered among patients with Parkinson's disease (PD), however, the pathophysiological mechanism remains unclear. Methods: We evaluated fatigue in 26 patients clinically diagnosed to have PD (16 men, 10 women) and age- and sex- matched 26 controls (16 men, 10 women) without neurological deficits by using a fatigue scale. In addition to neurological and neuropsychological examinations, all patients underwent MRI and SPECT using 99mTc-HMPAO. Results: Patients with PD had normal cognitive function as judged by the MMSE, but showed significantly high scores with the fatigue and depression scales in comparison to controls $(p<0.05)$. There was no significant correlation between the depression scale and the fatigue scale, or between the degree of disability and the fatigue scale in patients with PD, although a significant correlation existed between the depression scale and the fatigue scale in controls. With SPECT, a significant correlation was found between the fatigue scale and the reduction of perfusion in the frontal lobe $(p<0.05)$ in patients with PD. Conclusions: The present study suggested that sense of fatigue in patients with PD might be associated with frontal lobe dysfunction.
\end{abstract}

Keywords: Parkinson's disease, fatigue, frontal lobe, SPECT, Wisconsin card sorting test (WCST)

\section{Introduction}

Although fatigue is a frequently encountered complaint in patients with Parkinson's disease (PD), its pathophysiological mechanism remains unclear $[6,8,9$, 12]. Fatigue may be a mental or physical problem and may be linked to depression. For example, fatigue in dialysis patients was correlated with depression but not

*Corresponding author: Dr. Kazuo Abe, D-4, Department of Neurology, Osaka University Graduate School of Medicine, 2-2, Yamadaoka, Suita, Osaka 565-0871, Japan. Tel.: +81 6879 3571; Fax: +81 6879 3579; E-Mail: abe@neurol.med.osaka-u.ac.jp. with anemia. On the other hand, fatigue in patients with multiple sclerosis was not correlated with depression but correlated with cerebral dysfunction [11]. Friedman observed many non-depressed patients with PD also to have significant complaints of fatigue [6]. Based on these findings and our own clinical observations, we hypothesized that fatigue in patients with PD might be related to cerebral dysfunction, especially the frontal lobe dysfunction. To test this hypothesis, we sought a correlation between the severity of fatigue by using a questionnaire sheet and the frontal lobe function by using neuropsychological testing. We also evaluated those patients with single photon emission computed tomography (SPECT) to seek the presence of impairment of focal cerebral perfusion among PD patients with fatigue.

\section{Methods}

Interviews were undertaken from 26 patients with PD (16 men, 10 women, mean age $63.4 \pm 11.0$ years (mean-standard deviation) and mean duration of PD $4.6 \pm 3.2$ years) and 26 age- and sex- matched controls (16 men, 10 women, mean age $61.8 \pm 12.0$ years) who had been diagnosed to have tension or migraine headaches without neurological deficits. All patients had PD satisfying the criteria by Calne et al. [2] and were examined at our neurological clinic between 1997 and 1998. All patients were taking antiparkinson medication but no antidepressant or anticholinergic drugs. After neurological and neuropsychological examinations, we assessed the degree of fatigue by asking those patients and controls to fill the fatigue scale questionnaire consisting of 30 items [6,9]. Each item was a statement about fatigue being rated from 1 to 7 . Patients received brief instructions on the forms and filled them out by themselves. Depression was evaluated by the self-assessed depression scale (SDS) of Zung for all patients and controls [16]. The severity of PD was evaluated by the Hoehn and Yahr Scale [8] and the unified Parkinson's disease rating scale (UPDRS) [4]. Cognitive function was screened by the Mini Mental State Examination (MMSE) [5] and the frontal lobe 


\section{Fatigue Scale}

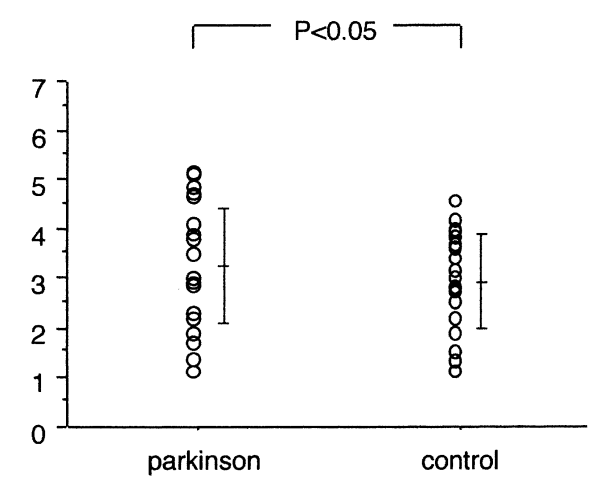

SDS

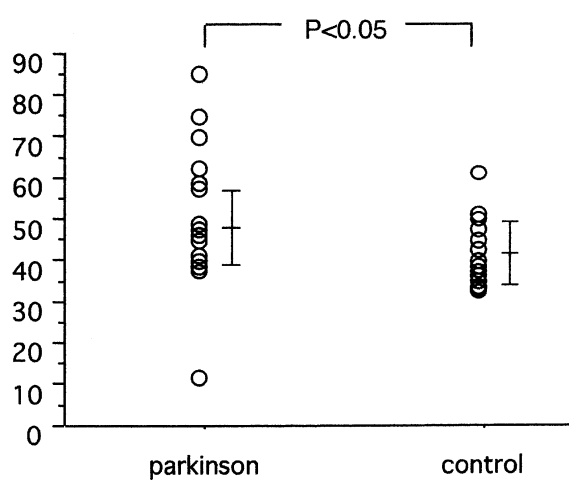

Fig. 1. Comparison of the fatigue scale and the self assessed depression scale. There were significant differences between patients with Parkinson's disease and controls both in the fatigue scale and the self assessed depression scale (SDS). The vertical bars denote mean \pm standard deviation.

Patients with Parkinson disease

\section{Fatigue Scale}

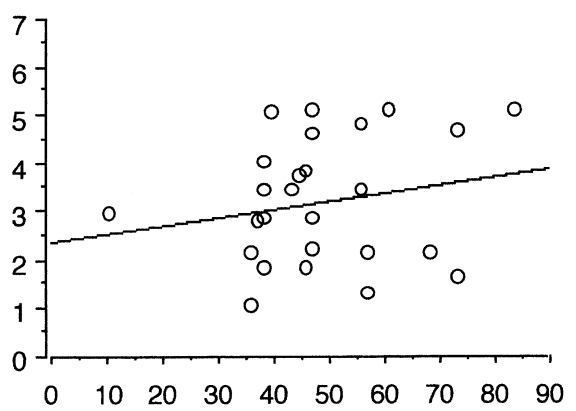

Depression Scale
Normal controls

\section{Fatigue Scale}

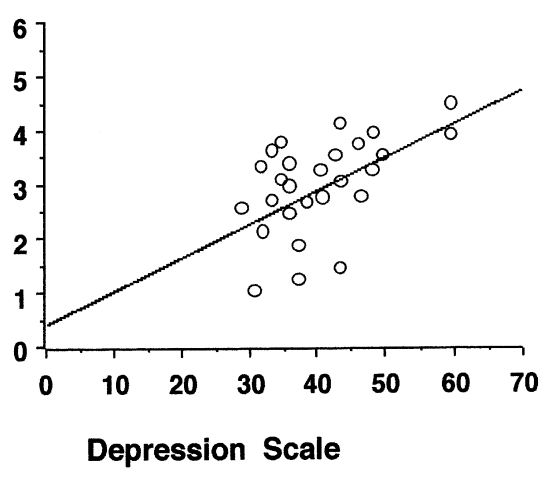

Fig. 2. Correlation between the fatigue Scale and the self assessed depression scale In patients with ParkinsonŠs disease, there was no significant correlation between the depression scale and the fatigue scale (left; $r=0.19, p>0.20)$. In controls, there was a significant correlation between the depression scale and the fatigue scale (right; $r=0.58, p<0.05$ ).

function was assessed by Go-No-Go task and the Wisconsin card sorting test (WCST) [7,11]. No attempt was made to control for medications during those tests. All patients and controls gave informed consent before those evaluations.

All patients were examined by cerebral magnetic resonance images (MRIs) to exclude cerebral abnormalities that might affect the clinical manifestations. The MRI studies were performed on a 1.5 Tesla device (Signa Advantage, General Electric Medical Systems, Milwaukee, WI) using a standard quadrature head coil. Single photon emission computed tomographic (SPECT) images were also obtained using technetium 99m d, 1-hexamethyl propyleneamine oxide (99mTc HMPAO) to evaluate cerebral perfusion. After acquisition of images, we set regions of interest (ROIs) in the frontal, temporal, parietal and occipital cortex as well as cerebellar hemispheres using transaxial images, and evaluated the presence of focal reduction of isotope uptake. ROIs in the cerebral cortexes were compared with those in the cerebellar hemispheres. The analysis of regional isotope uptake in a set of controls who had had tension headaches without organic brain diseases has shown the values of $82.0 \pm 7.0 \%$ in the frontal cortex, $80.5 \pm 7.8 \%$ in the temporal cortex, $79.9 \pm 8.0 \%$ in the parietal cortex, $85.8 \pm 6.2 \%$ in the occipital cortex as compared with uptakes in the cerebellar hemispheres. Thus, we have used the isotope uptake of a focal cerebral region below $50 \%$ of the maximal isotope uptake in the cerebellum to be abnormal after taking $95 \%$ confidence intervals into consideration [1]. All 
patients gave informed consent for MRI and SPECT examinations.

For statistical analysis, nonparametric MannWhitney's U-test was used to assess the differences between patients and normal. For correlation analysis, Spearman's rank correlation coefficient was used. All statistical analyses were carried out with a statistic software (STAT View 4.5-J, Hulinks Inc., Tokyo) using a microcomputer (Power Macintosh 8600/250, Apple computer Inc., Cupertino, CA). Statistical significance was defined as $p<0.05$.

\section{Results}

Patients with PD showed normal cognitive function as assessed by the MMSE (mean $28.1 \pm 1.6$, range $25-$ 30 ), but showed significantly higher scores on the fatigue scale $(3.4 \pm 1.2$ vs $2.9 \pm 0.9, p<0.05$, Fig. 1 left $)$ and the SDS $(47.0 \pm 9.2$ vs $40.4 \pm 8.4, p<0.05$, Fig. 1 right) in comparison to controls. Among patients with PD, there was no significant correlation between the SDS and the fatigue scale (Fig. 2), or between disability as judged by the Hoehn-Yahr scale/UPDRS scale and the fatigue scale, whereas there was a significant correlation between the SDS and the fatigue scale in controls ( $p<0.02$, Fig. 2$)$, even though the mean scores for the SDS and fatigue scales were lower than those in patients with PD (Fig. 1). With SPECT, there was a significant difference in the fatigue scale between patients with and without reduced uptake in the frontal lobe ( $3.7 \pm 1.1$ vs $2.2 \pm 0.8, p<0.05$, Fig. 3,4), but no significant difference was found in isotope uptakes in other regions of interest in compaison to the corresponding values obtained from patients with tension headache without organic brain diseases. Patients who achieved lower numbers of categories with the WCST tended to have reduction of isotope uptake in the frontal lobe with SPECT $(3.5 \pm 1.1$ vs $5.1 \pm 0.9, p=0.09$, Fig. 5). There was no difference in the Go-No-Go task between patients with PD and controls.

\section{Discussion}

In daily practice, we frequently encounter complaints of easy fatigue and exercise intolerance among patients with PD. In a previous study with questionnaire, $58 \%$ of patients with PD stated fatigue as one of the three most disabling symptoms, and $67 \%$ of them indicated that it differed from fatigue experienced be-

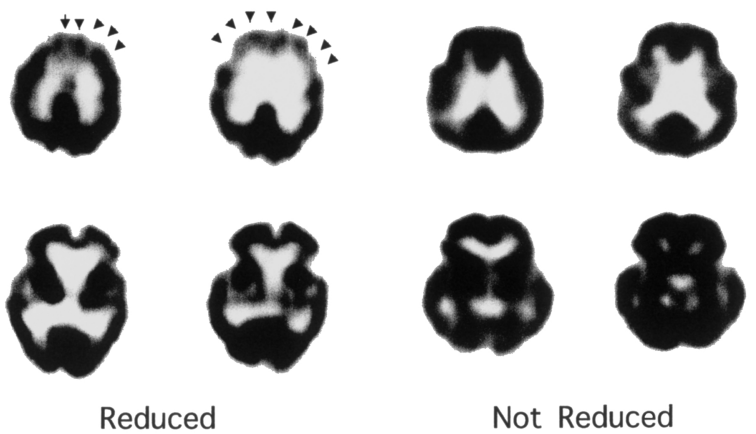

Fig. 3. Single photon emission computed tomography A representative patient with a high fatigue scale (fatigue score 5.1) showed significantly reduced isotope uptake in the frontal lobe (left), but another representative patient with a low fatigue scale (fatigue score 1.7) did not (right).

\section{Fatigue Scale}

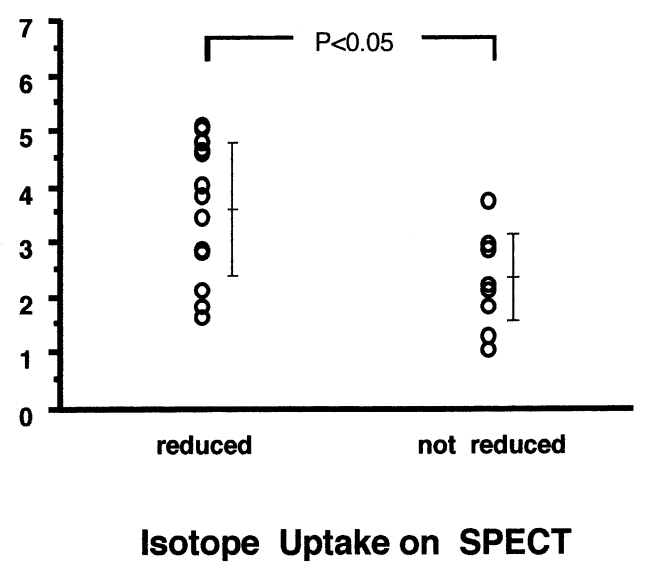

Fig. 4. The fatigue scale and the reduction of isotope uptake in the frontal lobe Patients with the reduction of isotope uptake in the frontal lobe on SPECT showed significantly higher fatigue scales. The vertical bars denote mean \pm standard deviation.

fore the onset of PD. [6] In the present study, we also found that patients with PD complained of fatigue more often than controls did. Despite its frequent encounter and functional importance, fatigue has not been investigated thoroughly in patients with PD.

Depression could be one of the identifiable causes of fatigue $[6,8,14,15]$. However, we found no correlation between the severity of depression and the fatigue scale in patients with PD, although the SDS scores were higher than those in controls. This suggested the presence of other factors in addition to depression. We did not control medication and this might have caused some confounding effects. However, the patients enrolled in this study took the same kind of antiparkinson drugs. We found a significant correlation between fa- 
Archieved Categories on WCST

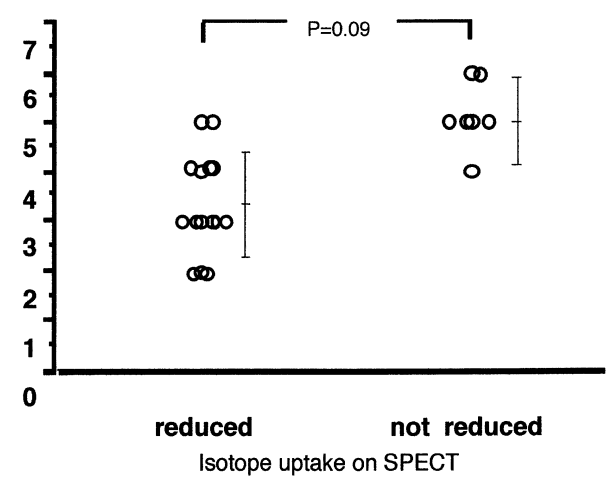

Fig. 5. The number of achieved categories on the Wisconsin Card Sorting Test and the reduction of isotope uptake There was a trend that the patients with the reduction in isotope uptake in the frontal lobe on SPECT achieved fewer categories on the Wisconsin Card Sorting Test. The vertical bars denote mean \pm standard deviation.

tigue and the reduction of isotope uptake in the frontal lobe and a trend of correlation between lower numbers of categories achieved with the WCST and fatigue. Roelcke et al. stated that the lesions in the premotor area participating in motor programming and executive function might contribute to the presence of fatigue [12]. Since deficits in motor programming and executive function are reflected in alternating and sequential tasks as well as in changing tasks demands in tests like the WCST [3], lower numbers of categories achieved with the WCST in our patients with PD suggested the presence of dysfunction in the premotor area. Using positron emission computed tomography, Roelcke et al. suggested that fatigue in multiple sclerosis was associated with dysfunction in the frontal cortex and basal ganglia as the result of demyelination in the frontal white matter [12]. Patients with PD should have dysfunction in the basal ganglia by the nature of the disease, but we found no significant correlation between the severity of fatigue and the severity of disability in the present study. This suggested that dysfunction in the basal ganglia might not be a cardinal contributor to fatigue. Based on these observations, we believe that frontal lobe dysfunction contributed to the sense of fatigue in patients with PD and eliminated positive correlation between the fatigue scale and the SDS, even though those patients had higher scores with the SDS as compared to controls.

\section{References}

[1] K. Abe, H. Fujimura, K. Toyooka, T. Hazama and N. Hirono et al., Single photon emission tomographic investigation of patients with motor neuron disease, Neurology 43 (1993), 1569-1573.

[2] D.B. Calne, B.J. Snow and C. Lee, Criteria for diagnosing Parkinson's disease, Ann Neurol 32 (1992), S125-S127.

[3] J.L. Cummings, Frontal-subcortical circuits and human behaviour, Arch Neurol 50 (1993), 873-880.

[4] S. Fahn and R.L. Elton, the UPDRS Development Committee. Unified Parkinson's Disease Rating Scale, in: Recent developments in Parkinson's disease, S. Fahn, C.D. Marsden, D.B. Calne and A. Lieberman, eds., MacMillan Health Care Information, Florham Park, NJ, 1987, pp. 153-163.

[5] M.F. Folstein, S.E. Folstein and P.R. Mchugh, Mini-Mental State Examination: a practical method for grading the mental state of patients for clinician, JPsychiatr Res 12 (1975), 189198.

[6] J. Friedman and H. Friedman, Fatigue in Parkinson's disease, Neurology 43 (1993), 2016-2018.

[7] R.K. Heaton, Wisconsin Card Sorting Test Manual, Psychological Assessment Resources, Oddessa, Fla, 1981.

[8] M.N. Hoehn and M.D. Yahr, Parkinsonism: onset, progression and mortality, Neurology 17 (1967), 427-442.

[9] L.B. Krupp, N.G. LaRocca, J. Muir-Nash and A.D. Steinberg, The fatigue severity scale: application to patients with multiple sclerosis and systemic lupus erythematosis, Arch Neurol $\mathbf{4 6}$ (1989), 1121-1123.

[10] L.B. Krupp and D.A. Pollina, Mechanisms and management of fatigue in progressive neurological disorders, Curr Opin Neurol 9 (1996), 456-460.

[11] M.E. Leimkuhler and M.-M. Mesulam, Reversible go-no go deficits in a case of frontal lobe tumor, Ann Neurol 18 (1985), 617-619.

[12] U. Roelcke, L. Kappos, H. Lechner-Scott, Brunnscweiler and $\mathrm{S}$. Huber et al., Reduced glucose metabolism in the frontal cortex and basal ganglia of multiple sclerosis patients with fatigue: An 18F-fluorodeoxyglucose positron emission tomography study, Neurology 48 (1997), 1566-1571.

[13] L. Sudarski, Central mechanism of fatigue, in: Chronic fatigue syndrome, D.M. Dawson and T.D. Sabin, eds., Little Brown, Boston, 1993, pp. 106-114.

[14] G.E. Tzelepis, F.D. McCool, J.H. Friedman and F.G. Jr. Hoppin, Respiratory muscle dysfunction in Parkinson's disease, Ann Rev Respir Dis 138 (1988), 266-271.

[15] I. Ziv, M. Avraham, Y. Michaelov and R. Djaldetti et al., Enhanced fatigue during motor performance in patients with Parkinson's disease, Neurology 51 (1998), 1583-1586.

[16] W.W.K.Zung, A self-rating depression scale, Archives of General Psychiatry 12 (1965), 63-70. 


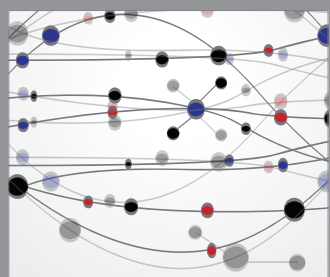

The Scientific World Journal
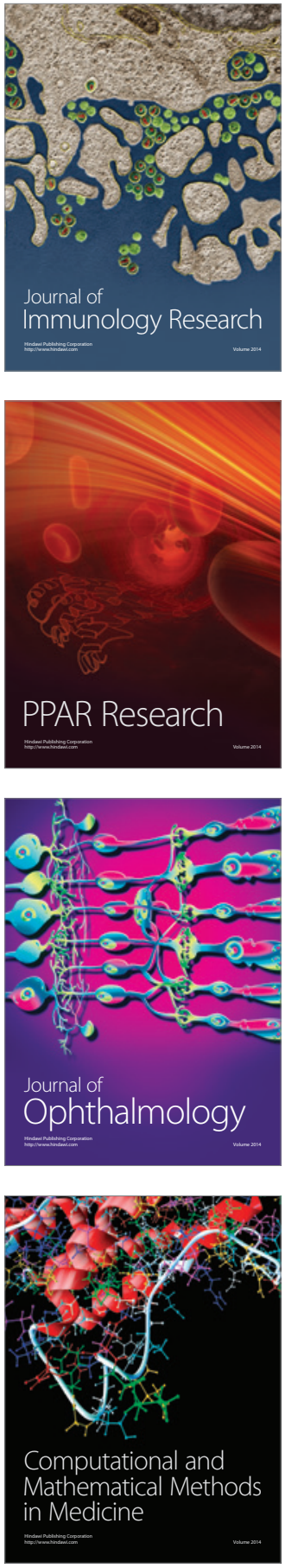

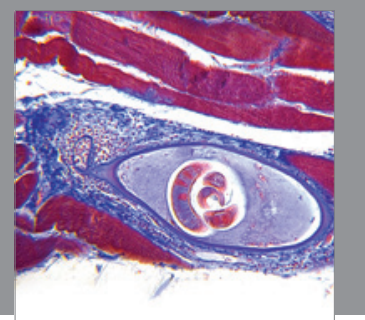

Gastroenterology

Research and Practice
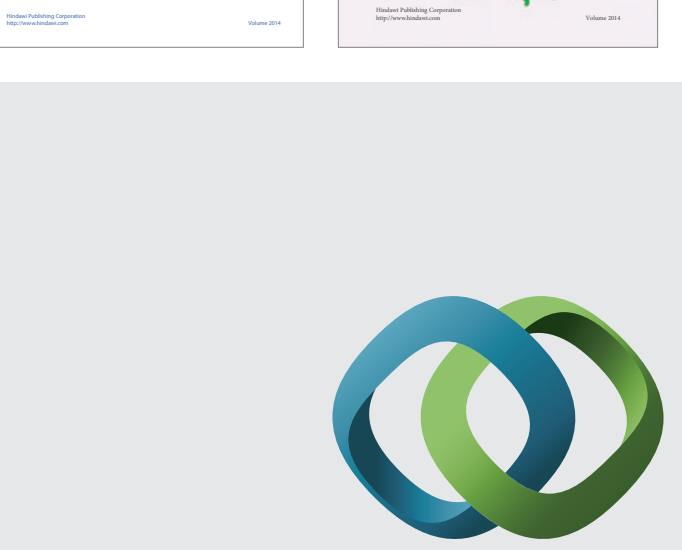

\section{Hindawi}

Submit your manuscripts at

http://www.hindawi.com
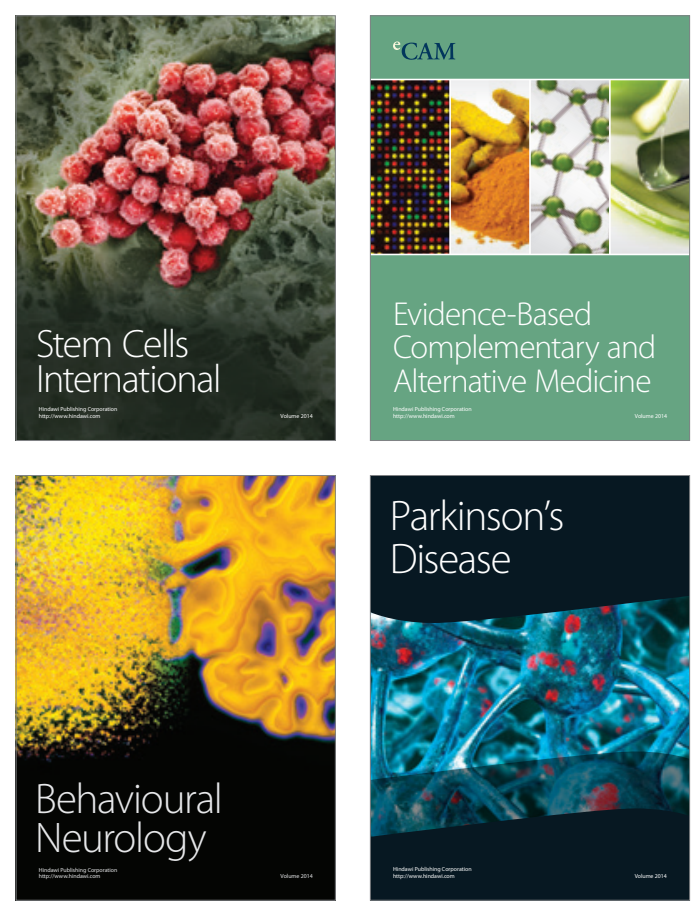

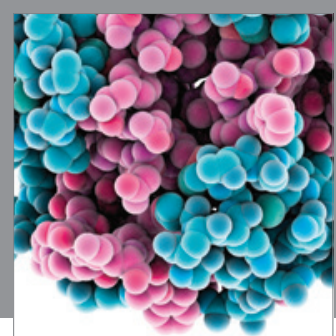

Journal of
Diabetes Research

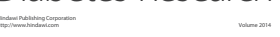

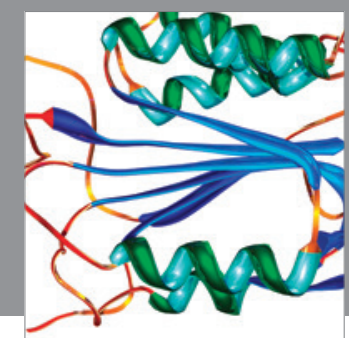

Disease Markers
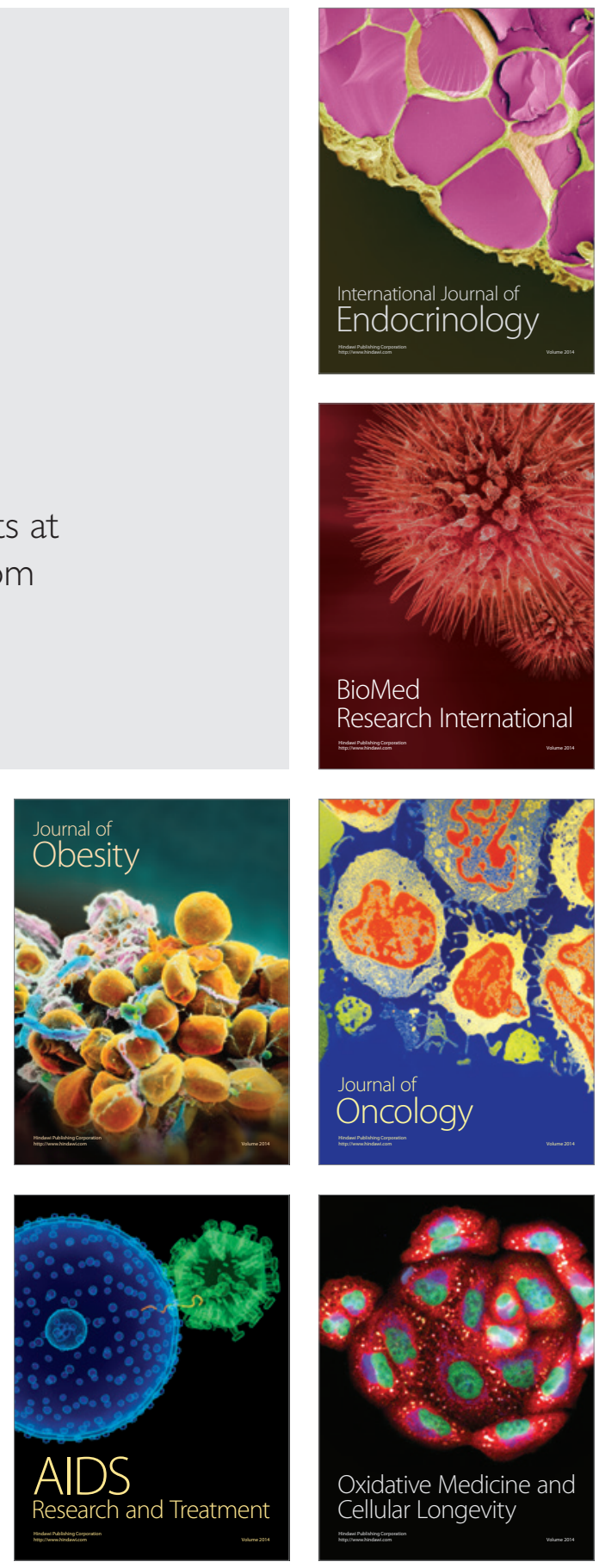